\title{
Health beliefs in oral cancer: Malaysian estate Indian scenario
}

Type:

Article

Abstract:

This is a study to describe the health beliefs related to oral cancer $(O C)$ in a high-risk group in Malaysia, a predominantly Indian community living in an agricultural setting called an estate. The study population was a convenient sample of 112 adults, above 20 years of age, attending oral cancer screening in two estates. The subjects consisted of $106(94.6 \%)$ Indians and six (5.4\%) Malays. Using the Health Belief Model, the perceptions of susceptibility to OC, its severity, and the benefits of and barriers to preventive actions, as well as beliefs underlying $\mathrm{OC}$ aetiology were investigated. About half of the subjects $(n=57,50.9 \%)$ felt susceptible to oral cancer. A majority of subjects $(\mathrm{n}=93,83.0 \%)$ felt that oral cancer is a severe disease. Thirty four people $(30.4 \%)$ perceived OC as a preventable disease, while $56(50 \%)$ did not, and the remaining 22 $(19.6 \%)$ did not know if OC was preventable or not. The majority of subjects (84.8\%) believed that modifications to the betel quid habit could be beneficial. The information solicited can be used as a starting point to design health-education activities aimed at this group in particular and the population in general. (C) 2001 Elsevier Science Ireland Ltd. All rights reserved.

\begin{tabular}{|c|l|}
\hline Author & $\begin{array}{l}\quad \text { Tan, B. S. } \\
\text { Ng, K. H. }\end{array}$ \\
& $\quad$ Esa, R. \\
\hline Source & Patient Education and Counseling \\
\hline ISSN & $0738-3991$ \\
\hline DOI & $10.1016 /$ s0738-3991(00)00121-x \\
\hline Volume (Issue) & $42(3)$ \\
\hline Page & $205-211$ \\
\hline Year & 2001 \\
\hline
\end{tabular}

Keyword:

health beliefs, oral cancer, high-risk community, Health beliefs, High-risk community. Oral cancer, alcohol, adult, aged, article, betel nut, cancer screening, cancer susceptibility, Community, female ,habit, health education, high risk population, human,Indian,major clinical study, Malaysia, male, mouth cancer, priority journal, tobacco, Aged, 80 and over, Areca, Culture, Follow-Up Studies, Health Knowledge, Attitudes, Practice, Humans, India, Middle Aged, Mouth Neoplasms 
Please Cite As:

TAN, B. S., NG, K. H. \& ESA, R. 2001. Health beliefs in oral cancer: Malaysian estate Indian scenario. Patient Education and Counseling, 42, 205-211

URL:

- http://apps.webofknowledge.com search via Accession No >>000166726900001

- http://www.scopus.com/inward/record.url?eid=2-s2.00035164248\&partner|D=40\&md5=81e5cdcc $1101 \mathrm{~b} 62230456411404207 \mathrm{cb}$

- http://mouthcancermalaysia.um.edu.my/abstract5.html

- http://www.ncbi.nlm.nih.gov/pubmed/11164319 